\title{
Solitary and Periodic Wave Solutions of the Fourth Order Boussinesq Equation Through the Novel Exponential Expansion Method
}

\author{
Ayrin Aktar*, Md Mashiur Rahhman, Kamalesh Chandra Roy \\ Department of Mathematics, Begum Rokeya University, Rangpur, Bangladesh \\ Email address: \\ ayrin.amath.ru@gmail.com (A. Aktar) \\ ${ }^{*}$ Corresponding author \\ To cite this article: \\ Ayrin Aktar, Md Mashiur Rahhman, Kamalesh Chandra Roy. Solitary and Periodic Wave Solutions of the Fourth Order Boussinesq Equation \\ Through the Novel Exponential Expansion Method. "merican Journal of "pplied Mathematics. Vol. 7, No. 2, 2019, pp. 50-58. \\ doi: 10.11648/j.ajam.20190702.12
}

Received: April 21, 2019; Accepted: June 13, 2019; Published: June 27, 2019

\begin{abstract}
This article presents the new exact traveling wave solutions of fourth order (1+1)-dimensional Boussinesq equation. We proposed a new exponential expansion method and apply to undertake this study. The analytical solutions are defined by various types of mathematical functions. This study further shows some solitary and periodic waves graphically. This paper also shows that the novel exponential expansion method is easily applicable and powerful mathematical tool in the symbolic computational approach in the field of mathematical physics and engineering. The exact solutions of this equation play a vital role for describing different types of wave propagation in any varied natural instances, especially in water wave dynamics.
\end{abstract}

Keywords: Novel Exponential Expansion Method, Boussinesq Equation, Solitary Wave Solutions, Periodic Solutions

\section{Introduction}

The nonlinear partial differential equations (NPDEs) are broadly used as models for describing various types of physical mechanisms of natural phenomena in the field of applied sciences and engineering, especially in plasma physics, elastic media, optical fibers, fluid dynamics, quantum mechanics, chimerical physics, biotechnology, signal processing, solid state physics, shallow water wave theory etc. The solitary and periodic wave solutions of these equations have importance physical significance to observe the oscillatory behaviors in these relevant fields. In addition, there are several types of mathematical method have been used to search the exact traveling wave solutions of NPDEs, such as the $\left(G^{\prime} / G\right)$-expansion method $[1,2]$, new approach of $\left(\mathrm{G}^{\prime} / \mathrm{G}\right)$-expansion method [3], novel $\left(\mathrm{G}^{\prime} / \mathrm{G}\right)$-expansion method [4], new approach of generalized $\left(\mathrm{G}^{\prime} / \mathrm{G}\right)$-expansion method [5], the tanh method [6-9], the Jacobi elliptic function method $[10,11]$, the homogeneous balance method [12-14], the Hirota's method [15], the Homotpy perturbation technique [16], the improved F-expansion method [17], the sine-cosine method [18], the modified simple equation method [19], the Exp-function method [20, 21], the exp (-Ф $(\xi)$ )-expansion method [22-26] and others.

The fourth order $(1+1)$-dimensional Boussinesq equation is an important class of NPDEs, which was first introduced by Boussinesq to examine the propagation of long waves in shallow water under the gravity propagating in positive and negative directions [27]. This equation also appeared as a model equation to describe the propagation of many other physical phenomena, such as iron sound wave in plasma, nonlinear lattice waves and vibrations in a nonlinear string. It was also applied to the study of the percolation of water in porous subsurface strata. There is an amount of literature, where the Boussinesq equation is well studied. For instance, Yildrim and Mohud-Din have been applied the He's semiinverse method to obtain the soliton solution of good Boussinesq equation [28]. Neyrame el al. have been examined the exact traveling wave solutions of the Boussinesq equation using the $\left(G^{\prime} / G\right)$-expansion method and the exact traveling wave solutions are expressed by the hyperbolic, trigonometric and rational functions with the help of auxiliary differential equation $G^{\prime \prime}+\lambda G^{\prime}+\mu G=0, \lambda, \mu \in \mathfrak{R}$ [29]. Alam et al. have 
been proposed a new method namely novel $\left(G^{\prime} / G\right)$-expansion method and its applied to find the more explicit solutions of this equation [4]. Many other well known methods, such as the inverse scattering transform method, the bilinear formalism, Hirota's method, tanh-coth method and Backlund transformation method have been used to handle completely integrable Boussinesq equation. Recently Akbar and Ali also studied the solitary wave solutions of the fourth order

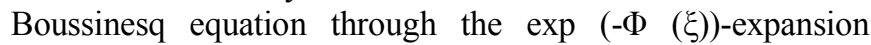
method [24]. They are provided only few solutions to this equation according to the nonlinear ordinary differential equation $\Phi^{\prime}(\xi)=\exp (-\Phi(\xi))+\mu \exp (\Phi(\xi))+\lambda$. In this regards, we are extended the $\exp (-\Phi(\xi))$-expansion method for searching more valuable explicit form solutions to NPDEs. The extended method is called an novel exponential expansion method and provides some new explicit and exact traveling wave solutions to the nonlinear evolution equations with help of subsidiary nonlinear ordinary differential equations $\Phi^{\prime}(\xi)+\sqrt{\lambda+\mu(\exp (-\Phi(\xi)))^{2}}=0 \quad$ and $\quad \Phi^{\prime}(\xi)+$ $\lambda \exp (\Phi(\xi))+\mu \exp (-\Phi(\xi))=0$. The aim of this article is to discover for new study linking to the novel exponential expansion method for solving the famous nonlinear fourth order Boussinesq equation to demonstrate the correctness and truthfulness of the method. The exp $(-\Phi(\xi))$-expansion method is only special case of the novel exponential expansion method. It is shown that this method provides some new explicit solutions. The exact traveling wave solutions are obtained according to the variation of the additional unknown parameters that are involved in explicit solutions. The exact solutions have its great importance to reveal the internal mechanism of the physical phenomena. Algebraic manipulation of the technique with the help of Maple is much easier than the other methods.

The remainder of the paper is organized as follows: In Section 2, we give the brief description of the proposed novel exponential expansion method. In Section 3, we apply this method to investigate the explicit and solitary wave solutions of the Boussinesq equation for describing various types of wave propagation in any varied physical instances. The physical explanations of the obtaining solutions and the advantages of the new exponential expansion method are presented in section 4 and 5 respectively. Conclusions are given in the last section.

\section{Description of the Proposed Novel Exponential Expansion Method}

This section presents the brief descriptions of the new exponential expansion method.

Let us consider the nonlinear PDE as:

$$
f\left(u, u_{t}, u_{x}, u_{x x}, u_{t t}, u_{t x}, \ldots\right)=0
$$

where, $f$ is a function of $u, u_{t}, u_{x}, u_{x x}, u_{t t}, u_{t x}, \ldots$ and the subscripts denote the partial derivatives of $u(x, t)$ with respect to $x$ and $t$.

Let $u(x, t)=u(\xi), \xi=x \pm V t$, where $V$ is an arbitrary constant, then the eq. (1) reduces to a nonlinear ordinary differential equation (ODE) for $u=u(\xi)$ :

$$
g\left(u, u^{\prime}, u^{\prime \prime}, u^{\prime \prime \prime}, \cdots\right)=0
$$

where, $g$ is a function of $u, u^{\prime}, u^{\prime \prime}, u^{\prime \prime \prime}, \cdots$ and its derivatives point out the ordinary derivatives with respect to $\xi$.

Let us consider the traveling wave solution of eq. (4) as:

$$
u(\xi)=\sum_{i=0}^{N} A_{i}(\exp (-\Phi(\xi)))^{i}, A_{N} \neq 0
$$

where the coefficients $A_{i}(0 \leq i \leq N)$ are constants to be evaluated and $\Phi=\Phi(\xi)$ satisfies the first order nonlinear ordinary differential equation:

$$
\Phi^{\prime}(\xi)=\exp (-\Phi(\xi))+\mu \exp (\Phi(\xi))+\lambda
$$

where $\lambda$ and $\mu$ are arbitrary constants.

The value of the positive integer $N$ appeared in equation (3) can be determined by balancing the higher order derivative with the nonlinear terms of the highest order appeared in equation (2).

By substituting (3) into (2) and using (4) rapidly, we obtain a system of algebraic equations for $A_{i}(0 \leq i \leq N), \lambda, \mu, V$. With the help of symbolic computation, such as Maple, we can evaluate the obtaining system and find out the values $A_{i}(0 \leq i \leq N), \lambda, \mu, V$. It is notable that equation (4) has the following five types of general solutions [22-26]:

$$
\begin{gathered}
\Phi(\xi)=\ln \left(\frac{-\sqrt{\Theta} \tanh \left\{0.5 \sqrt{\Theta}\left(\xi+\xi_{0}\right)\right\}-\lambda}{2 \mu}\right), \mu \neq 0, \Theta=\lambda^{2}-4 \mu>0 \\
\Phi(\xi)=\ln \left(\frac{\sqrt{-\Theta} \tan \left\{0.5 \sqrt{-\Theta}\left(\xi+\xi_{0}\right)\right\}-\lambda}{2 \mu}\right), \mu \neq 0, \Theta=\lambda^{2}-4 \mu>0 \\
\Phi(\xi)=-\ln \left(\frac{\lambda}{\exp \left(\lambda\left(\xi+\xi_{0}\right)\right)-1}\right), \mu=0, \lambda \neq 0, \Theta=\lambda^{2}-4 \mu>0 \\
\Phi(\xi)=\ln \left(-\frac{2\left(\lambda\left(\xi+\xi_{0}\right)+2\right)}{\lambda^{2}\left(\xi+\xi_{0}\right)}\right), \mu \neq 0, \lambda \neq 0, \Theta=\lambda^{2}-4 \mu=0 \\
\Phi(\xi)=\ln \left(\xi+\xi_{0}\right), \lambda=0, \mu=0
\end{gathered}
$$

where $\xi_{0}$ is the integrating constant

Thus the multiple explicit solutions of NPDE (1) are obtained by using of the equations (3) and (5)-(9).

Again, suppose eq. (2) has solution of the form (3) and $\Phi=\Phi(\xi)$ satisfies another first order nonlinear ODE:

$$
\Phi^{\prime}(\xi)+\sqrt{\lambda+\mu(\exp (-\Phi(\xi)))^{2}}=0, \lambda, \mu \in \mathfrak{R}
$$

By substituting (3) into (2) and using (10) repeatedly, we obtain a system of algebraic equations for $A_{i}(0 \leq i \leq$ 
$N), \lambda, \mu, V$. With the help of symbolic computation, such as Maple, we can evaluate the resulting system and find out the values $A_{i}(0 \leq i \leq N), \lambda, \mu, V$. It is notable that equation (10) has the following general solutions:

$$
\begin{gathered}
\Phi(\xi)=-\ln \left(-\sqrt{\frac{\lambda}{\mu}} \operatorname{csch}\left[\sqrt{\lambda}\left(\xi+\xi_{0}\right)\right]\right), \lambda>0, \mu>0 \\
\Phi(\xi)=-\ln \left(\sqrt{\frac{-\lambda}{\mu}} \sec \left[\sqrt{-\lambda}\left(\xi+\xi_{0}\right)\right]\right), \lambda<0, \mu>0 \\
\Phi(\xi)=-\ln \left(\sqrt{\frac{\lambda}{-\mu}} \sec \left[\sqrt{\lambda}\left(\xi+\xi_{0}\right)\right]\right), \lambda>0, \mu<0 \\
\Phi(\xi)=-\ln \left(\sqrt{\frac{-\lambda}{\mu}} \csc \left[\sqrt{-\lambda}\left(\xi+\xi_{0}\right)\right]\right), \lambda<0, \mu>0 \\
\Phi(\xi)=-\ln \left(\frac{1}{ \pm \sqrt{\mu}\left(\xi+\xi_{0}\right)}\right), \lambda=0, \mu>0 \\
\Phi(\xi)=-\ln \left(\frac{i}{ \pm \sqrt{\mu}\left(\xi+\xi_{0}\right)}\right), \lambda=0, \mu<0
\end{gathered}
$$

where $\xi_{0}$ is the integrating constant.

Thus the new multiple exact solutions of NPDE (1) are obtained by combining the equations (3) and (11)-(16).

Finally, suppose equation (2) has solution of the form (3) and $\Phi=\Phi(\xi)$ satisfies another first order nonlinear ODE:

$$
\Phi^{\prime}(\xi)+\lambda \exp (\Phi(\xi))+\mu \exp (-\Phi(\xi))=0, \lambda, \mu \in \Re
$$

By substituting (3) into (2) and using (17) repeatedly, we obtain a system of algebraic equations for $A_{i}(0 \leq i \leq$ $N), \lambda, \mu, V$. With the help of symbolic computation, such as Maple, we can calculate the resulting system and find out the values $A_{i}(0 \leq i \leq N), \lambda, \mu, V$. It is notable that equation (17) has the following general solutions:

$$
\begin{gathered}
\Phi(\xi)=-\ln \left(\sqrt{\frac{\lambda}{\mu}} \tan \left[\sqrt{\lambda \mu}\left(\xi+\xi_{0}\right)\right]\right), \lambda \mu>0 \\
\Phi(\xi)=-\ln \left(-\sqrt{\frac{\lambda}{\mu}} \cot \left[\sqrt{\lambda \mu}\left(\xi+\xi_{0}\right)\right]\right), \lambda \mu>0 \\
\Phi(\xi)=-\ln \left(\sqrt{\frac{\lambda}{-\mu}} \tanh \left[\sqrt{-\lambda \mu}\left(\xi+\xi_{0}\right)\right]\right), \lambda \mu<0 \\
\Phi(\xi)=-\ln \left(\sqrt{\frac{\lambda}{-\mu}} \operatorname{coth}\left[\sqrt{-\lambda \mu}\left(\xi+\xi_{0}\right)\right]\right), \lambda \mu<0 \\
\Phi(\xi)=-\ln \left(-\frac{1}{\mu\left(\xi+\xi_{0}\right)}\right), \lambda=0, \mu>0
\end{gathered}
$$

where $\xi_{0}$ is the integrating constant and $\lambda \mu>0$ or $\lambda \mu<0$ are dependent on sign of $\lambda$.

Finally, we obtain the new multiple explicit solutions of NPDE (1) by combining the equations (3) and (18)-(22).

\section{Applications of the Method}

This section presents the application of the proposed new exponential expansion method to find the more explicit and exact traveling wave solutions of the fourth order Boussinesq equation.

The fourth order $(1+1)$-dimensional Boussinesq equation is given by

$$
u_{t t}-u_{x x}-u_{x x x x}-3\left(u^{2}\right)_{x x}=0
$$

Here, $u(x, t)$ represents the evolution of free surface of the fluid and the subscripts denotes partial derivatives. Boussinesq was first introduced the equation (23) as a model equation to investigate the propagation of long waves in shallow water under the gravity propagating in both directions. This equation also helpful to observe the wave propagation of water in porous subsurface strata, the vibration of the string, the nonlinear lattice waves, the iron sound waves in plasma or at any varied instances where the model equation (23) is applicable. Equation (23) possesses solitary waves, take out from traveling wave solutions and Boussinesq was first who gave s scientific explanation of their existence. In order to get the new traveling wave solutions, if we introduce the wave variable transformation $u(x, t)=u(\eta), \eta=x-V t$, where $V$ is the speed of traveling, then the equation (23) reduces to a nonlinear ODE as:

$$
\left(V^{2}-1\right) \frac{d^{2} u}{d \eta^{2}}-\frac{d^{4} u}{d \eta^{4}}-3 \frac{d^{2}}{d \eta^{2}}\left(u^{2}\right)=0
$$

Integrating (11) twice with regard to $\eta$, we get:

$$
\left(V^{2}-1\right) u-\frac{d^{2} u}{d \eta^{2}}-3 u^{2}+C=0
$$

where $C$ is an integral constant.

It is easily shown that, the pole of equation (25) is $N=2$. According to the novel exponential expansion method, the solution of the equation (25) can be written as:

$$
u(\xi)=A_{0}+A_{1} e^{-\Phi(\eta)}+A_{2}\left(e^{-\Phi(\eta)}\right)^{2}
$$

where $\Phi(\eta)$ satisfies the nonlinear ODEs (4), (10) and (17).

Akbar and Ali have been investigated the explicit and exact solitary wave solutions according to the first order nonlinear ODE (4) [24]. So, we have no need to analyze the solutions according to the old $\exp (-\Phi(\xi))$-expansion method. We are only analyzed the exact solutions of (25) according to the auxiliary nonlinear ODEs (10) and (17).

Now, substituting (26) into (25) and using (10) rapidly, we obtain a system of algebraic equation by equating the coefficients of $\left(e^{-\Phi(\eta)}\right)^{i},(i=0,1,2,3,5)$ to zero as follows:

$$
\left.\begin{array}{l}
V^{2} A_{0}-3 A_{0}^{2}-A_{0}+C=0, \\
-A_{1} \lambda+V^{2} A_{2}-6 A_{0} A_{1}-A_{1}=0, \\
-3 A_{1}^{2}+V^{2} A_{2}-4 A_{2} \lambda-6 A_{0} A_{2}-A_{2}=0 \\
-2 A_{1} \mu-6 A_{1} A_{2}=0,-6 A_{2} \mu-3 A_{2}^{2}=0 .
\end{array}\right\}
$$

Solving the system of algebraic equation (27), we have 


$$
\left\{C=-\frac{1}{12} V^{4}+\frac{1}{6} V^{2}+\frac{4}{3} \lambda^{2}-\frac{1}{12}, V=V, A_{0}=\frac{1}{6} V^{2}-\frac{2}{3} \lambda-\frac{1}{6}, A_{1}=0, A_{2}=-2 \mu\right\}
$$

where $V, \lambda$ and $\mu$ are arbitrary constants.

By using the equations (11)-(16), (26) and (28), the Boussinesq equation (23) has the following new explicit solutions:

$$
\begin{gathered}
u_{1}(x, t)=\frac{1}{6} V^{2}-\frac{2}{3} \lambda-\frac{1}{6}-2 \lambda \operatorname{csch}^{2}\left[\sqrt{\lambda}\left(x-V t+\xi_{0}\right)\right], \lambda>0, \mu>0 \\
u_{2}(x, t)=\frac{1}{6} V^{2}-\frac{2}{3} \lambda-\frac{1}{6}-2 \lambda \operatorname{sech}^{2}\left[\sqrt{\lambda}\left(x-V t+\xi_{0}\right)\right], \lambda>0, \mu<0 \\
u_{3}(x, t)=\frac{1}{6} V^{2}-\frac{2}{3} \lambda-\frac{1}{6}-2 \lambda \csc ^{2}\left[\sqrt{-\lambda}\left(x-V t+\xi_{0}\right)\right], \lambda<0, \mu>0 \\
u_{4}(x, t)=\frac{1}{6} V^{2}-\frac{2}{3} \lambda-\frac{1}{6}-2 \lambda \sec ^{2}\left[\sqrt{-\lambda}\left(x-V t+\xi_{0}\right)\right], \lambda<0, \mu>0 \\
u_{5}(x, t)=\frac{1}{6} V^{2}-\frac{1}{6} \mp 2 \mu\left(\frac{1}{x-V t+\xi_{0}}\right)^{2}, \lambda=0, \mu>0
\end{gathered}
$$

Now, substituting (26) into (25) and using (17) rapidly, we obtain a system of algebraic equation by equating the coefficients of $\left(e^{-\Phi(\eta)}\right)^{i},(i=0,1,2,3,5,6)$ to zero as follows:

$$
\left.\begin{array}{l}
-6 A_{2} \mu \lambda+C+V^{2} A_{0}-3 A_{0}^{2}-A_{0}=0, \\
-6 A_{0} A_{1}+V^{2} A_{1}-A_{1}-2 A_{1} \mu^{2}=0, \\
-6 A_{0} A_{2}-3 A_{1}^{2}-A_{2}+2 A_{2} \lambda^{2}-6 A_{2} \mu^{2}+V^{2} A_{2}=0, \\
-2 A_{2} \mu \lambda-3 A_{2}^{2}=0,-6 A_{1} A_{2}=0,-2 A_{1} \mu \lambda=0 .
\end{array}\right\}
$$

Solving the system of algebraic equation (34), we have:

$$
\left\{\begin{array}{c}
C=-2 \mu^{2} \lambda^{2}+\frac{1}{6} V^{2}-\frac{1}{12} V^{4}-\frac{1}{12}+\frac{1}{3} \lambda^{4}+3 \mu^{4}, V=V, A_{0}=-\frac{1}{6}-\frac{1}{3} \lambda^{2}-\mu^{2}+\frac{1}{6} V^{2} \\
\mu=\mu, \lambda=\lambda, A_{1}=0, A_{2}=-\frac{2}{3} \mu \lambda
\end{array}\right\}
$$

where $V, \lambda$ and $\mu$ are arbitrary constants.

Using the equations (18)-(22), (26) and (35), the fourth order Boussinesq equation (23) gives the following new explicit solutions:

$$
\begin{gathered}
u_{6}(x, t)=-\frac{1}{6}-\frac{1}{3} \lambda^{2}-\mu^{2}+\frac{1}{6} V^{2}-\frac{2}{3} \lambda^{2} \tan ^{2}\left[\sqrt{\lambda \mu}\left(x-V t+\xi_{0}\right)\right] \lambda \mu>0 \\
u_{7}=-\frac{1}{6}-\frac{1}{3} \lambda^{2}-\mu^{2}+\frac{1}{6} V^{2}-\frac{2}{3} \lambda^{2} \cot ^{2}\left[\sqrt{\lambda \mu}\left(x-V t+\xi_{0}\right)\right] \lambda \mu>0 \\
u_{8}(x, t)=-\frac{1}{6}-\frac{1}{3} \lambda^{2}-\mu^{2}+\frac{1}{6} V^{2}-\frac{2}{3} \lambda^{2} \tanh ^{2}\left[\sqrt{-\lambda \mu}\left(x-V t+\xi_{0}\right)\right] \lambda \mu<0 \\
u_{9}(x, t)=-\frac{1}{6}-\frac{1}{3} \lambda^{2}-\mu^{2}+\frac{1}{6} V^{2}-\frac{2}{3} \lambda^{2} \operatorname{coth}^{2}\left[\sqrt{-\lambda \mu}\left(x-V t+\xi_{0}\right)\right] \lambda \mu<0
\end{gathered}
$$

The explicit solutions obtained in this article are encouraging, applicable and could be useful to describe the long wave translation on the surface of a fluid layer under the action of gravity, the propagation of iron sound waves in plasma, the propagation of nonlinear lattice waves and the propagation of vibrations in a nonlinear string. The solutions are also applied to study linking to the percolation of water in porous subsurface strata.

\section{Physical Explanation}

This section presents the physical importance of the fourth order Boussinesq equation and physical significances of the above determined solutions.
The fourth order Boussinesq equation that contains nonlinear term and dispersive terms, describes the various types of wave translations in shallow water under the gravity propagating in both directions, plasma physics, nonlinear lattice and nonlinear string. This equation incorporates two competing effects: (i) the nonlinear term represented by $\left(u^{2}\right)_{x x}$ that describe the translations of wave and (ii) the linear dispersion term represented by $u_{x x x x}$ that describes the spreads it out. If both dispersion and nonlinearity are present, solitary waves can be persistent. Hence it is notable to point out that the delicate balance between the nonlinearity effect of $\left(u^{2}\right)_{x x}$ and the dissipative effect of $u_{x x x x}$ to the Boussinesq equation give rise to solitary waves. A solitary wave is a wave which propagates without any temporal 
evolution in shape or size when viewed in the reference frame moving with the group velocity of the wave. The envelope of the wave has one global peak and decays far away from the peak. Solitary waves arise in many literatures, including the elevation of the surface of water, the intensity of light in optical fibers, the particle wave propagation in field theory, the elevation of surface in shallow water wave etc. A soliton is also a nonlinear solitary wave with the additional property that the wave retains its permanent structure, even after interacting with another soliton. For example, two solitons propagating in opposite directions effectively pass through each other without breaking. Sometimes nonlinearity without dispersion prevents the formation of solitary waves, because the pulse energy is frequently pumped into higher frequency modes. Similarly to dispersion, dissipation can also give rise to solitary waves when combined with nonlinearity. There are various types of solitary and periodic wave solutions that appeared from the analytical solutions to the nonlinear evolution equation by choosing appropriate values of the physical parameters. In this article, the solitary wave and periodic wave solutions originated from the explicit solutions to the Boussinesq equation for some special values of additional free parameters as follows:

Solution $u_{1}(x, t)$ corresponding to the fixed values $V=1, \lambda=0.5, \mu=0.5$, and $\xi_{0}=0.5$ represent the single soliton type solitary wave solution in negative direction, which is shown in Figure 1. Solution $u_{2}(x, t)$ represent the non-topological bell nature solitary wave in negative direction with fixed parameters $V=1, \lambda=0.1, \mu=1$, and $\xi_{0}=0.5$, which is shown in Figure 2. Solution $u_{3}(x, t)$ with fixed parameters $V=1, \lambda=-0.1, \mu=0.1$ and $\xi_{0}=0.5$ is presented periodic traveling wave solution, which is shown in Figure 3. Solution $u_{4}(x, t)$ with fixed parameters $V=$ $1, \lambda=-1, \mu=1$, and $\xi_{0}=0.5$ is represented the soliton type periodic traveling wave solution, which is shown in Figure 4. Solution $u_{5}(x, t)$ is formed the solitary wave solutions of singular soliton type corresponding to the fixed values $V=$ $0.5, \lambda=0, \mu=0.5$ and $\xi_{0}=0$, which is shown in Figure 5. Solution $u_{6}(x, t)$ with fixed parameters $V=1, \lambda=1, \mu=1$ and $\xi_{0}=0.5$ is formed the soliton type periodic traveling wave solution in negative direction, which is shown in Figure 6. Solution $u_{7}(x, t)$ with fixed parameters $V=1, \lambda=0.5, \mu=$ 0.5 and $\xi_{0}=0$ is formed the singular periodic traveling wave solution, which is shown in Figure 7. Solution $u_{8}(x, t)$ is presented the solitary wave solutions of singular soliton type corresponding to $V=0.5, \lambda=1, \mu=-1$ and $\xi_{0}=0.5$, which is shown in Figure 8. Finally, solution $u_{9}(x, t)$ represent the solitary wave solution corresponding to $V=0.1, \lambda=-1.5, \mu=1.5$ and $\xi_{0}=0$, which is shown in Figure 9.

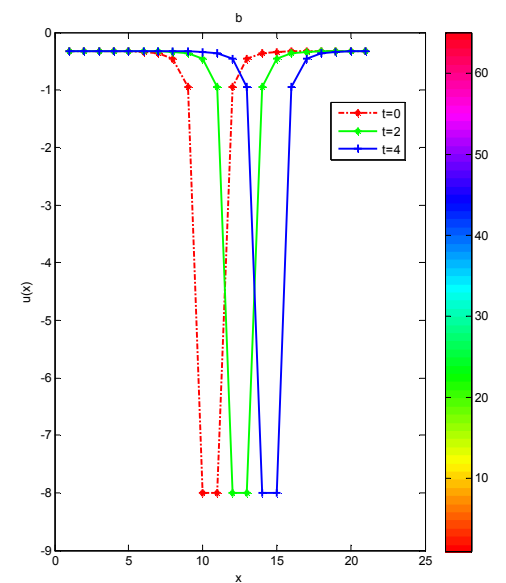

Figure 1. Solitary wave solution of single soliton nature in negative direction (a) $3 D$ plot of solution $u_{1}(x, t)$ and (b) $2 D$ plot solution $u_{1}(x, t)$ for $V=1, \lambda=$ $0.5, \mu=0.5$, and $\xi_{0}=0.5, t=0, t=2$ and $t=4$.
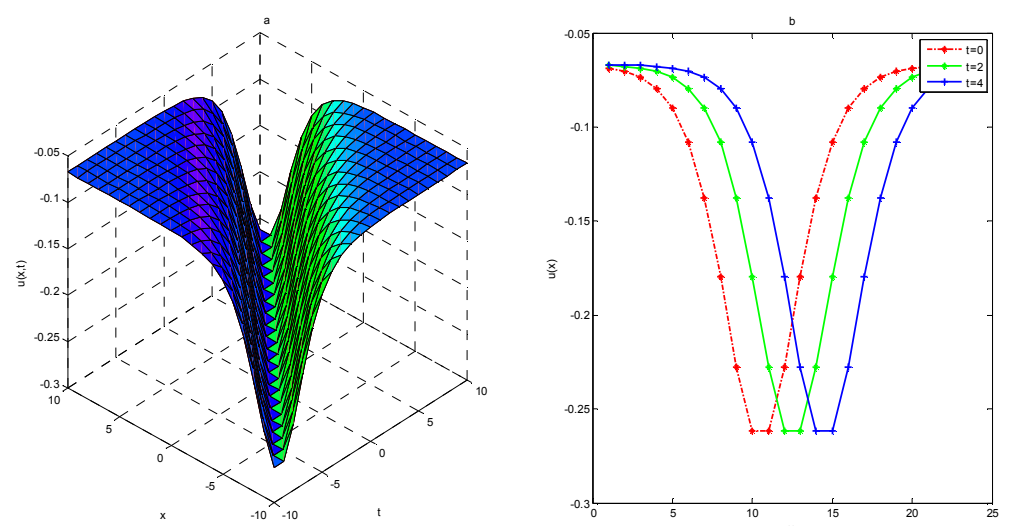

Figure 2. Solitary wave solution of non-topological bell nature in negative direction (a) $3 D$ plot of solution $u_{2}(x, t)$ and (b) $2 D$ plot solution $u_{2}(x, t)$ for $V=1, \lambda=0.1, \mu=1$, and $\xi_{0}=0.5, t=0, t=2$ and $t=4$. 

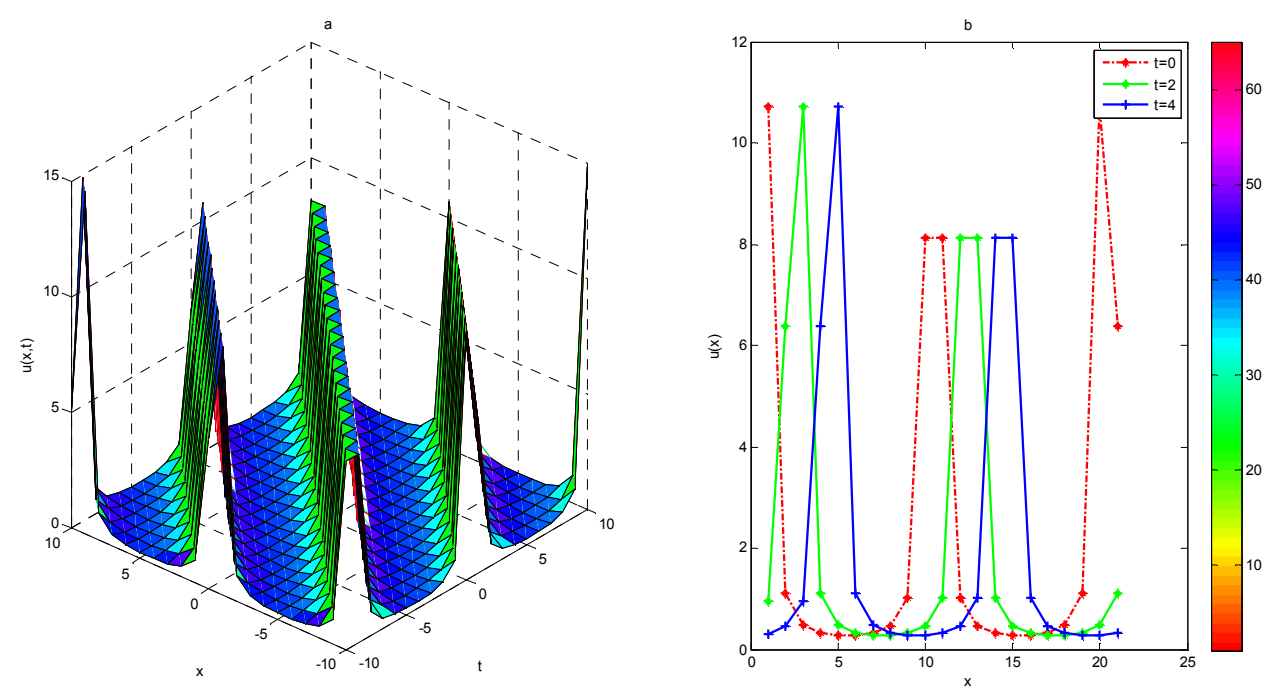

Figure 3. Periodic wave solution in positive direction (a) $3 D$ plot of solution $u_{3}(x, t)$ and (b) $2 D$ plot solution $u_{3}(x, t)$ for $V=1, \lambda=-0.1, \mu=0.1$ and $\xi_{0}=0.5, t=0, t=2$ and $t=4$.
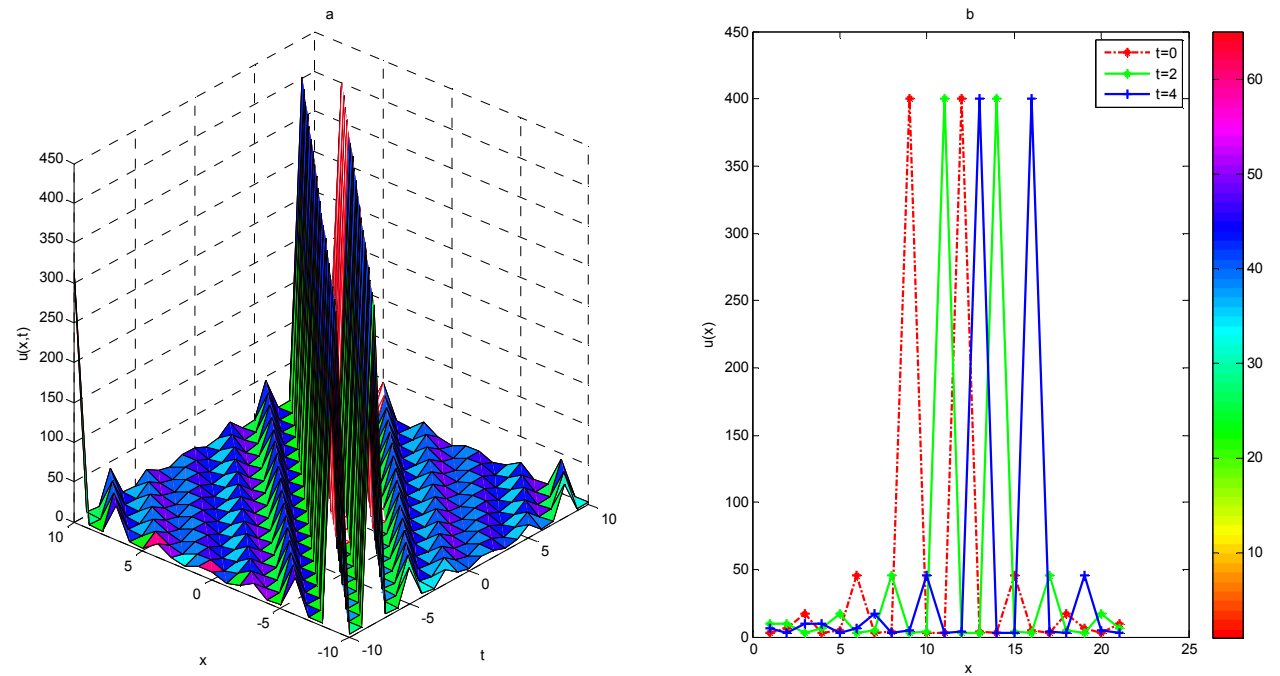

Figure 4. Periodic wave solution of soliton nature in positive direction (a) $3 D$ plot of solution $u_{4}(x, t)$ and (b) $2 D$ plot solution $u_{4}(x, t)$ for $V=1, \lambda=-1, \mu=$ 1 , and $\xi_{0}=0.5, t=0, t=2$ and $t=4$.
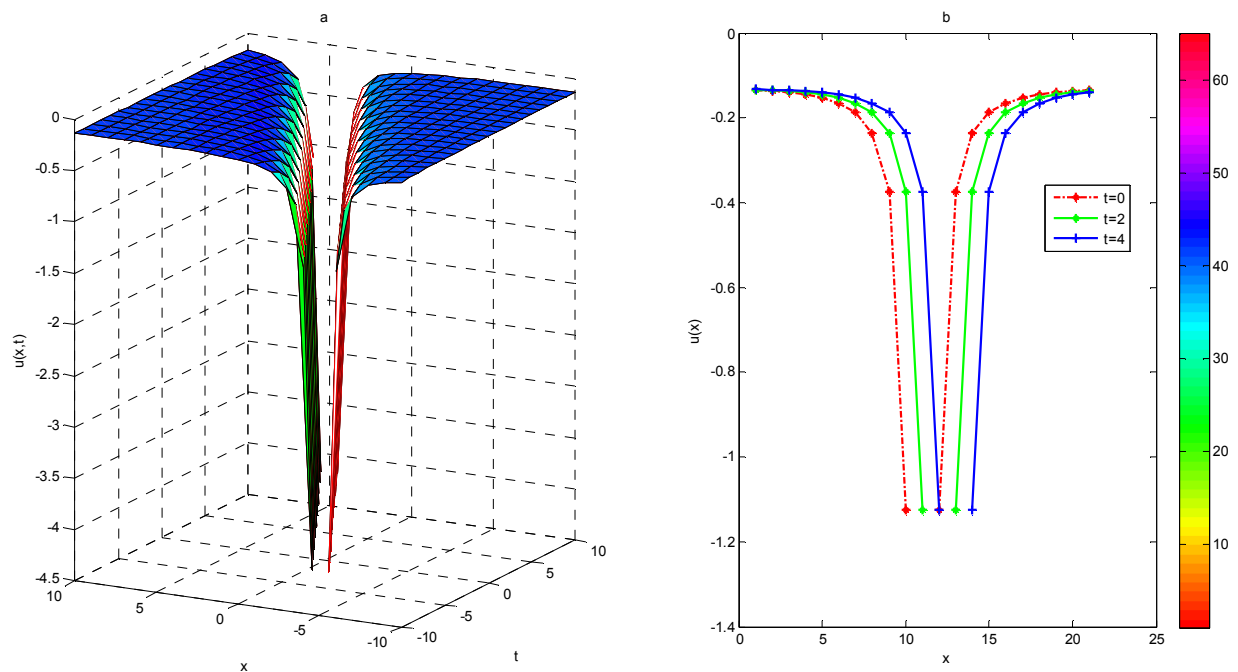

Figure 5. Solitary wave of singular soliton nature in negative direction (a) $3 D$ plot of solution $u_{5}(x, t)$ and (b) $2 D$ plot solution $u_{5}(x, t)$ for values $V=$ $0.5, \lambda=0, \mu=0.5$ and $\xi_{0}=0, t=0, t=2$ and $t=4$. 

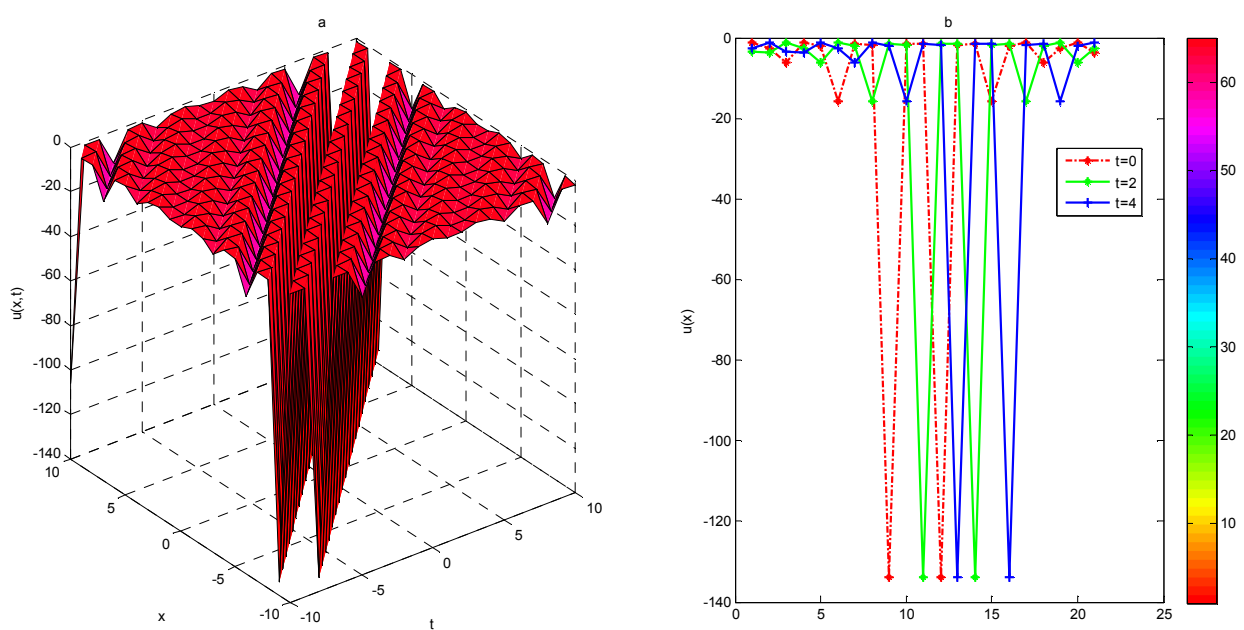

Figure 6. Periodic wave solution of soliton nature in negative direction (a) $3 D$ plot of solution $u_{6}(x, t)$ and (b) $2 D$ plot solution $u_{6}(x, t)$ for $V=1, \lambda=1, \mu=$ 1 and $\xi_{0}=0.5, t=0, t=2$ and $t=4$.
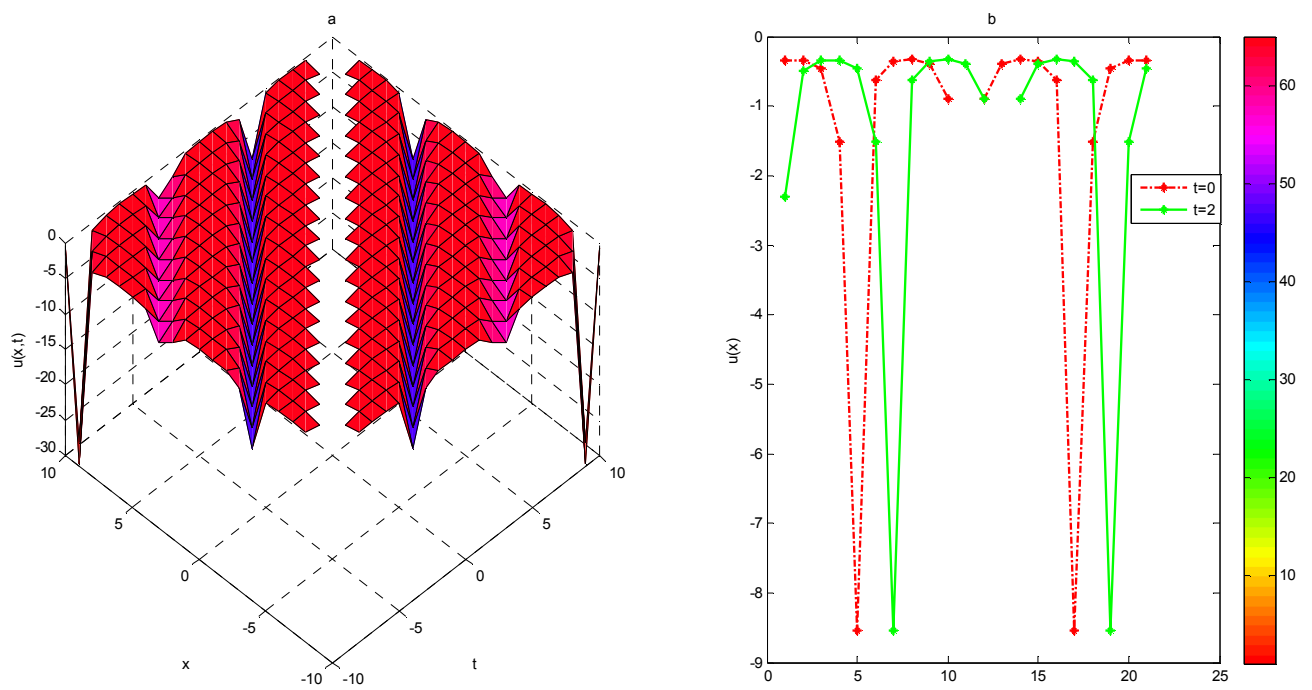

Figure 7. Singular periodic wave solution (a) $3 D$ plot of solution $u_{7}(x, t)$ and (b) $2 D$ plot solution $u_{7}(x, t)$ for $V=1, \lambda=0.5, \mu=0.5$ and $\xi_{0}=0, t=0, t=$ 2 and $t=4$.
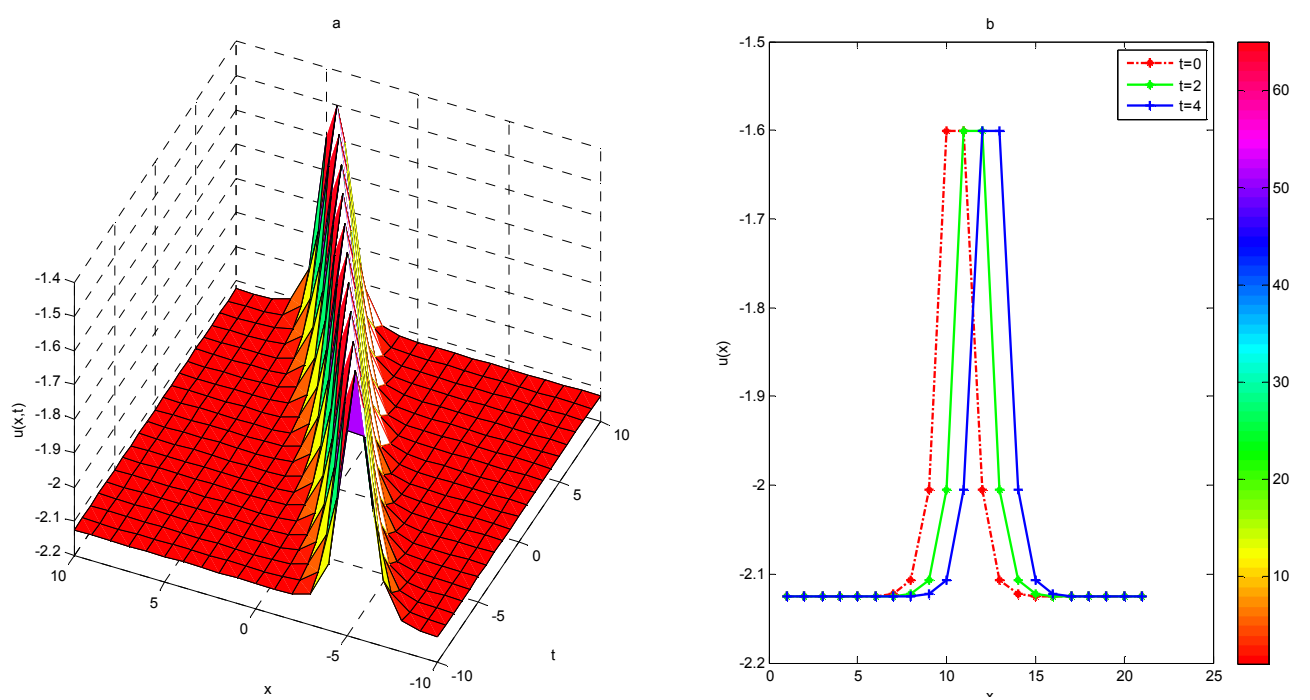

Figure 8. Solitary wave solution of cupson nature (a) $3 D$ plot of solution $u_{8}(x, t)$ and (b) $2 D$ plot solution $u_{8}(x, t)$ for $V=0.5, \lambda=1, \mu=-1$ and $\xi_{0}=0.5$, $t=0, t=2$ and $t=4$. 

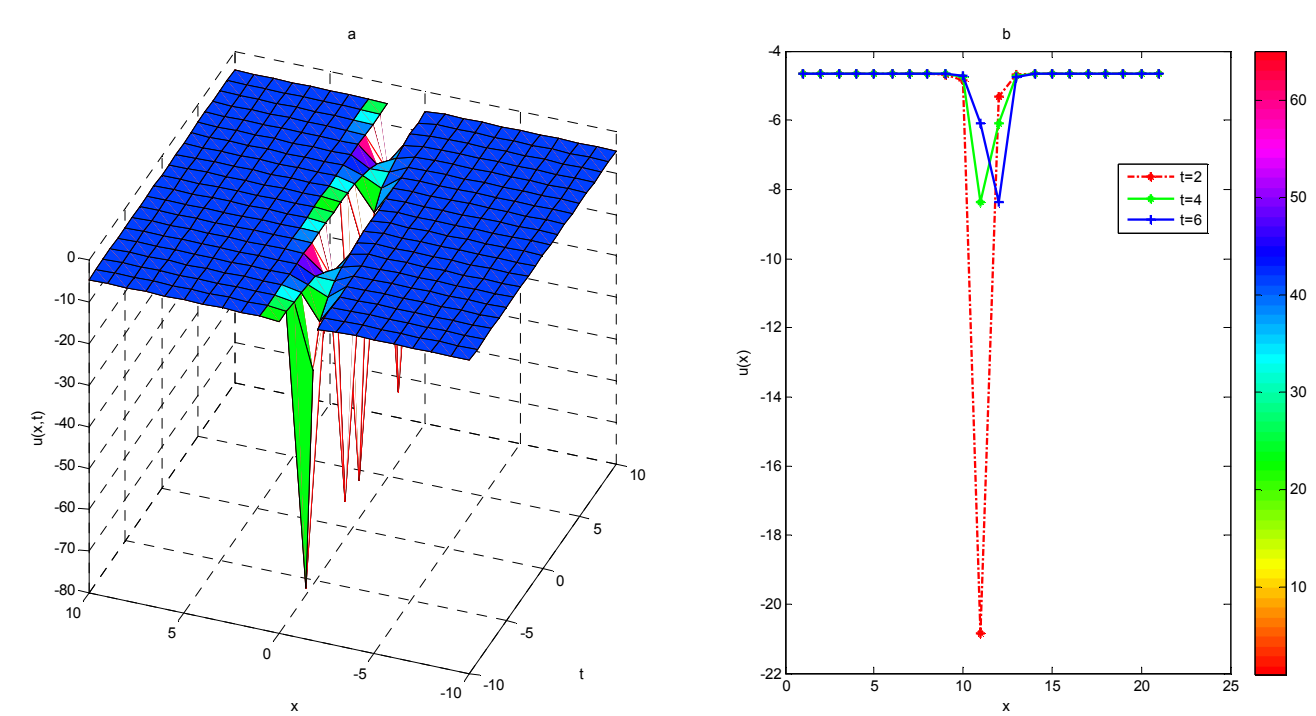

Figure 9. Solitary wave solution (a) $3 D$ plot of solution $u_{9}(x, t)$ and (b) $2 D$ plot solution $u_{9}(x, t)$ for $V=0.1, \lambda=-1.5, \mu=1.5$ and $\xi_{0}=0, t=0, t=2$ and $t=4$.

\section{Discussion}

The novel exponential expansion method has been given more general and huge amount of new explicit solutions with few additional free parameters. The explicit solutions represented several types of solitary and periodic wave solutions according to the variation of the unknown parameters. The wave solutions have its great importance to interpret the inner mechanism of the natural physical phenomena. The solitary and periodic wave solutions are also useful for verifying the accuracy and stability of popular numerical schemes such as finite element and finite difference methods. It is worth declaring that some of our obtained solutions are in good agreement with already published results. For instances, the solution $u(x, t)=$ $-2\left(\frac{C_{2}}{C_{2}(x-V t)+C_{1}}\right)^{2}+\frac{3 \lambda}{2}+a_{0}, \lambda^{2}-4 \mu=0$ of the Boussinesq equation found in [29] corresponding to fixed parametric values $\lambda=2, \mu=1, a_{0}=-3, V=1, C_{2}=1$ is equal to our obtained solution $\quad u_{5}(x, t)=\frac{1}{6} V^{2}-\frac{1}{6}-$ $2 \mu\left(\frac{1}{x-V t+\xi_{0}}\right)^{2}$ corresponding to the values $\mu=1, V=1$, $C_{1}=\xi_{0}$. The other obtained traveling waves solutions include in this paper are new and have not been found in previous literature $[4,29]$. Therefore, the novel exponential expansion method provides some new exact solutions which are not found in other literature. This is the main advantage of this method. This method not only re-derives all known solutions in a systemic way but also obtains several entirely new and more explicit solutions to the NPDEs. Algebraic manipulation of the method with the help of Maple is much easier than the other existing method. We have also presented some shapes of solitary waves constructed by choosing suitable values of the involved free parameters to visualize the underlying mechanism to the original physical phenomena. Using mathematical software MATHLAB, three and two dimensional plots of the determined solutions have been shown in Figures1 to 9 .

\section{Conclusions}

In this paper, we have proposed a new exponential expansion method for solving NPDEs and successfully applied to obtain more explicit and exact traveling wave solutions of the fourth order Boussinesq equation. The results revealed that some of the analytical solutions have obtained in the new forms. We have also formulated solitary wave solutions in graphically from the explicit solutions by taking appropriate parametric values of the additional free parameters. The paper has shown that the exponential expansion method is sufficient to search for more new exact solutions of NPDEs in mathematical physics and engineering. The obtained solutions can be utilized to further analyze by physicists or engineers on varied instances. It can be concluded that this method can be implemented to obtain useful solutions for other NPDEs.

\section{References}

[1] M. L. Wang, X. Z. Li, J. Zhang, The (GG)-expansion method and travelling wave solutions of nonlinear evolution equations in mathematical physics. Phys. Lett. A 372 (2008) 417.

[2] H. Kim, R. Sakthivel, New Exact Traveling Wave Solutions of Some Nonlinear Higher-Dimensional Physical Models. Reports Math. Phys. 70 (1) (2012) 39.

[3] H. Naher, F. A. Abdullah, New generalized and improved $\left(G^{\prime} / G\right)$-expansion method for nonlinear evolution equations in mathematical physics. AIP Advan. 3 (2013) 032116.

[4] M. N. Alam, M. A. Akbar, S. T. Mohyud-Din, A novel ( $\left.\mathrm{G}^{\prime} / \mathrm{G}\right)-$ expansion method and its application to the Boussinesq equation. Chin. Phys. B 23 (2) (2014) 020202.

[5] M. N. Alam, M. A. Akbar and H. O. Rohid, Traveling wave solutions of the Boussinesq equation via the new approach of generalized $\left(\mathrm{G}^{\prime} / \mathrm{G}\right)$-expansion method. SpringerPlus 3 (2014); 43. 
[6] W. Malfliet, Hereman W. The tanh method: I. Exact solutions of nonlinear evolution and wave equations. Physica. Scr. $1996 ; 54 ; 563-568$

[7] Wazwaz AM. The extended tanh method for new compact and noncompact solutions for the KP-BBM and the ZK-BBM equations. Chaos, Solitons Fract. 2008; 38 (5); 1505-1516.

[8] Abdou MA, Soliman AA. Modified extended tanh-function method and its application on nonlinear physical equations. Phys. Lett. A 2006; 353 (6); 487-492.

[9] El-Wakil SA, Abdou MA. New exact travelling wave solutions using modified extended tanh-function method. Chaos, Solitons Fract. 2007; 31 (4); 840-852.

[10] Liu S, Fu Z, Liu SD, Zhao Q. Jacobi elliptic function expansion method and periodic wave solutions of nonlinear wave equations. Phys. Lett. A 2001; 289; 69-74.

[11] Chen Y, Wang Q. Extended Jacobi elliptic function rational expansion method and abundant families of Jacobi elliptic functions solutions to $(1+1)$-dimensional dispersive long wave equation. Chaos, Solitons Fract. 2005; 24; 745-757.

[12] Zhao X, Tang D. A new note on a homogeneous balance method. Phys. Lett. A 2002; 297 (1-2); 59-67.

[13] Zhao X, Wang L, Sun W. The repeated homogeneous balance method and its applications to nonlinear partial differential equations. Chaos, Solitons Fract. 2006; 28 (2); 448-453.

[14] Zhaosheng F. Comment on "On the extended applications of homogeneous balance method. Appl. Math. Comput. 2004; 158 (2); 593-596.

[15] Hirota R. Exact solution of the KdV equation for multiple collisions of solutions. Phys. Rev. Lett. 1971; 27; 1192-1194.

[16] Chun C, Sakthivel R. Homotopy perturbation technique for solving two points boundary value problems -camparison with other mathods. Computer Phy. Commu. 2010; 181 (6); 1021-1024.

[17] Wang, Zhang HQ. Further improved F-expansion method and new exact solutions of Konopelchenko-Dubrovsky equation. Chaos Solitons Fract. 2005; 25; 601-610.

[18] Wazwaz AM. A sine-cosine method for handle nonlinear wave equations. App. Math. Comp. Model. 2004; 40; 499-508.
[19] Jawad AJM, Petkovic MD and Biswas A. Modified simple equation method for nonlinear evolution equations. Appl. Math. Comput., 2010; 217; 869-877.

[20] $\mathrm{He} \mathrm{JH}, \mathrm{Wu}$ XH. Exp-function method for nonlinear wave equations. Chaos, Solitons Fract. 2006; 30 (3); 700-708.

[21] Noor MA, Mohyud-Din ST, Waheed A. Exp-function method for travelling wave solutions of nonlinear evolution equations. Appl. Math. Comput. 2010; 216; 477-483.

[22] Hafez MG, Ali MY, Kauser MA, Akter MT. Application of the $\exp (-\Phi(\xi))$-expansion method to find exact solutions of the (1+1)-dimensional dispersive long wave equations. British J. Math. Com. Sci. 2014; 4 (22); 3191-3201. Doi: 10.9734/BJMCS/2014/13129.

[23] Hafez MG, Alam MN, Akbar MA. Traveling wave solutions for some important coupled nonlinear physical models via the coupled Higgs equation and the Maccari system. Journal of Kink Saud University.-Science (in press) 2014.

[24] Akbar MA, Ali NHM. Solitary wave solutions of the fourth order Boussinesq equation through the exp $(-\Phi(\xi))$-expansion method. Springer Plus 2014; 3: 343. doi: 10.1186/2193-18013-344

[25] Hafez MG, Kauser MA, Akter MT. Some new exact traveliing wave solutions of the cubic nonlinear Schrodinger equation using the $\exp (-\Phi(\xi))$-expansion method. International J. Sci. Eng. Tech. 2014; 3 (7); 848-851.

[26] Hafez MG, Kauser MA, Akter MT. Some new exact traveling wave solutions for the Zhiber-Shabat equation. British J. Math. Com. Sci. 2014; 4 (18); 2582-2593.

[27] Lai S, Wu YH, Zhou Y. Some physical structures for the (2+1)-dimensional Boussinesq water equation with positive and negative exponents. Comput Math Appl 2008; 56: 339 345 .

[28] Yildirm A, Mohyud-Din ST. A variational approach for soliton solution of good Boussinesq equation. J. King Saud Uni. (Sci.) 2010; 22; 205-208.

[29] Neyrame A, Roozi A, Hosseini S S and Shafiof S M. Exact traveling wave solutions for some nonlinear partial differential equations. J. King Saud Univ. (Sci.) 2012; 22: 275-278. 